\title{
SEARCHING FOR LIGHT ECHOES DUE TO CIRCUMSTELLAR MATTER IN SNe Ia SPECTRA
}

\author{
Sebastián Marino ${ }^{1,2}$, Santiago González-Gaitán ${ }^{1,2}$, Francisco Förster $^{2,3}$, Gastón Folatelli ${ }^{4,5}$, \\ Mario HAMUY ${ }^{1,2}$, AND ERIC HSIaO ${ }^{6,7}$ \\ ${ }^{1}$ Departamento de Astronomía, Universidad de Chile, 1515 Camino El Observatorio, Las Condes, Santiago, Chile \\ ${ }^{2}$ Millennium Institute of Astrophysics, Casilla 36-D, Santiago, Chile \\ ${ }^{3}$ Centro de Modelamiento Matemático, Universidad de Chile, Av. Blanco Encalada 2120 Piso 7, Santiago, Chile \\ ${ }^{4}$ Instituto de Astrofsica de La Plata (IALP, CONICET), Argentina \\ ${ }^{5}$ Kavli Institute for the Physics and Mathematics of the Universe (Kavli IPMU, WPI), \\ the University of Tokyo, Kashiwa 277-8583, Japan \\ ${ }^{6}$ Carnegie Observatories, Las Campanas Observatory, Casilla 601, La Serena, Chile \\ ${ }^{7}$ Department of Physics and Astronomy, Aarhus University, Ny Munkegade 120, DK-8000 Aarhus C, Denmark \\ Received 2014 November 14; accepted 2015 April 26; published 2015 June 12
}

\begin{abstract}
We present an analytical model for light echoes (LEs) coming from circumstellar material (CSM) around Type Ia Supernovae (SNe Ia). Using this model we find two spectral signatures at $4100 \AA$ and $6200 \AA$ that are useful to identify LEs during the Lira law phase (between 35 and 80 days after maximum light) coming from nearby CSM at distances of $0.01-0.25 \mathrm{pc}$. We analyze a sample of $89 \mathrm{SNe}$ Ia divided into two groups according to their $B-V$ decline rate during the Lira law phase, and search for LEs from CSM interaction in the group of SNe with steeper slopes by comparing their spectra with our LE model. We find that a model with LEs + pure extinction from interstellar material (ISM) fits the observed spectra better than a pure ISM extinction model that is constant in time, but we find that a decreasing extinction alone explains the observations better without the need of LEs, possibly implying dust sublimation due to the radiation from the SN.
\end{abstract}

Key words: circumstellar matter - galaxies: ISM - supernovae: general

\section{INTRODUCTION}

Type Ia supernovae (SNe Ia) are one of the most studied objects in astronomy. Owing to their standardizable high luminosities that make them unrivaled distance indicators up to high redshifts $(z \sim 2$, Jones et al. 2013), astronomers searching for the ultimate fate of the universe have studied them in ever greater detail, discovering several thousands of them up to now (e.g., Sako et al. 2014). Despite their success as cosmological probes, increasing observations and clues reveal that the puzzle of the nature and mechanism generating these colossal explosions is still far from reaching a conclusive solution.

As best shown in recent well-studied nearby objects (Nugent et al. 2011; Bloom et al. 2012), SNe Ia may originate from the explosion of a compact CO-rich white dwarf (WD) in a binary system. A common candidate considered for the binary companion has long been a non-degenerate star, such as a main-sequence or a red-giant star, that donates mass to the WD (single degenerate, SD, scenario) either in a stable fashion so that the WD nears the Chandrasekhar mass and explodes first subsonically and then supersonically (Ch-SD, e.g., Röpke et al. 2012; Blondin et al. 2013; Sim et al. 2013) or via unstable accretion leading to an initial detonation in the outer layer of the WD that triggers a subsequent detonation near the center prior to reaching the Chandrasekhar mass (sub Ch-SD, e.g., Kromer et al. 2010; Sim et al. 2012).

From an observational point of view, the SD scenario model has evidence both for and against. Among the observations that disfavor the model are: the absence of hydrogen and helium in their spectra (Lundqvist et al. 2013; Shappee et al. 2013); as well as the absence of radio and X-ray emission (Chomiuk et al. 2012; Horesh et al. 2012) which sets tight constraints on mass loss from a progenitor; the non-detection of early emission from shock interaction with a companion (Hayden et al. 2010; Bianco et al. 2011; Bloom et al. 2012) and the pre- explosion non-detections ( $\mathrm{Li}$ et al. 2011) that generally rule out red giants and He stars; the lack of sufficient galactic X-ray emission (Di Stefano 2010; Gilfanov \& Bogdán 2010) and UV radiation (Woods \& Gilfanov 2013; Johansson et al. 2014) expected from mass accretion in the Ch-SD scenario; and the measured SNe Ia rate as a function of redshift, which challenges the modeled delay time distribution for the classical Ch-SD scenario (Maoz \& Mannucci 2012, and references therein). Some of these issues can be addressed through different alternative SD models (Di Stefano et al. 2011; Justham 2011).

Among the observational evidence that favors the SD channel is the presence of nearby circumstellar material (CSM), presumably from mass loss in the progenitor system prior to explosion, manifested from: CSM/ejecta interaction (Hamuy et al. 2003; Silverman et al. 2013c), and most notably in the case of PTF11kx (Dilday et al. 2012); the discovery of narrow Na I D absorption lines that vary with time (Patat et al. 2007; Blondin et al. 2009; Simon et al. 2009; Sternberg et al. 2014); and the statistical preference for the interstellar lines to show blueshifts (Sternberg et al. 2011b; Maguire et al. 2013; Phillips et al. 2013). Altogether, the observational evidence suggests the possibility of multiple channels for SNe Ia.

Additionally, it has been suggested that such nearby CSM could affect the colors of SNe Ia through light scattering in the line of sight and explain in this way some of the differences in total-to-selective extinction ratios $\left(R_{V}\right)$ found in $\mathrm{SN}$ hosts compared to the Milky Way (MW; Wang 2005; Goobar 2008; Amanullah \& Goobar 2011). Heavily extincted SNe clearly show a different $R_{V}$ while $\mathrm{SNe}$ with moderate extinction show values consistent with the MW (e.g., Mandel et al. 2011; Burns et al. 2014). Cosmological studies using standard light-curve fitters obtain a luminosity-color relation that suggests reddening laws lower than the MW (e.g., Guy et al. 2005), but it is possible that SN intrinsic colors are more complicated and 
incorrectly modeled (e.g., Conley et al. 2007; Chotard et al. 2011; Scolnic et al. 2014).

Understanding the origin of the dispersion of $\mathrm{SNe}$ Ia colors not only affords the opportunity to understand their nature, but also to remove a major source of systematic uncertainty in SNe Ia cosmology. As shown in Kim et al. (2013), color might indeed be the principal parameter of diversity in SNe Ia light curves, followed only by the well known light-curve width parameter of SNe Ia. Förster et al. (2013, hereafter F13) showed that the evolution of SNe Ia color with time is related to the strength of the narrow sodium absorption, suggesting that at least some part of it might originate from a closer interaction with dust than with the host interstellar medium (ISM). In particular, they found that redder objects at maximum light have stronger narrow absorption lines and evolve faster from red to blue during the late time evolution of the Lira law decline of 30-90 days past maximum light (Lira 1995; Phillips et al. 1999). Possible explanations for this are light echoes (LEs) from CSM that affect the late-time colors or, alternatively, CSM dust sublimation (DS) in the line of sight.

In this paper, we aim to investigate the results of F13 further and test the hypothesis of nearby CSM by looking for spectroscopic signatures of LEs in a large sample of nearby $\mathrm{SNe}$ Ia spectra in the Lira law phase. Echoes from nearby ISM (and possible CSM) have previously been reported for SN 1991T, SN 1998bu, SN 2006X, SN 1992G, and SN 2014J at nebular phases (Schmidt et al. 1994; Cappellaro et al. 2001; Wang et al. 2008a; Silverman et al. 2013a; Crotts 2015). These individual studies focused on echoes generated at large distances from the $\mathrm{SN}$, tens to hundreds of parsecs away, scattering hundreds of days past maximum light.

Here we search for LEs at earlier times ( $>30$ days past maximum light), coming from nearby CSM dust that is at less than a parsec from the SN. Such CSM can potentially affect the colors and the Lira law decline rate (Amanullah \& Goobar 2011). Hence we search for LEs in the group of SNe analyzed in F13 that presented more extinction and a steeper than normal $B-V$ evolution (hereafter fast Lira decliners) to test the hypothesis that these may originate in regions of nearby CSM. To do this, we use SNe with a shallow Lira law slope (hereafter slow Lira decliners) as a reference set of $\mathrm{SNe}$ without CSM interaction.

In Section 2 we present our simple LE model. Then in Section 3 we focus on the prediction of observable spectroscopy features to look for LEs. In Section 4 we present the data and the analysis. Section 5 summarizes the results of our search for LEs and in Section 6 we discuss the success of our LE model, the validity of our assumptions, and other possible mechanisms that could explain fast Lira decliners. Finally, the main conclusions are summarized in Section 7.

\section{LE MODEL}

The effects of the interaction of light with intervening dust from CSM causing scattering away from the line of sight, and therefore extinction and reddening, have been studied and modeled in depth in the past (e.g., Chevalier 1986; Wang 2005; Patat 2005; Patat et al. 2006; Goobar 2008). We present here a simple analytical model that is easy to implement numerically and makes clear observable predictions to directly compare with data. The CSM consists of a simple spherically isotropic shell of dust (with $R_{V}=3.1$ ) that absorbs and scatters the light of the $\mathrm{SN}$. The radius of the shell is initially fixed at $0.05 \mathrm{pc}$ to produce LEs reaching the observer with time delays of $\sim 50$ days and affecting the colors during the Lira law phase, as light emitted at maximum light is observed at later epochs. At these distances we expect the temperature of the dust to be slightly lower than the sublimation temperature $(2000 \mathrm{~K})$. We only consider single scattering for simplicity and because multiple scattering in the CSM becomes important when its optical depth is larger than 1 (see Patat 2005). According to our analysis all the SNe we considered have a total optical depth $\lesssim 1$ in the visible, with the exception of SN 1997cw, SN $1999 \mathrm{gd}, \mathrm{SN} 2003 \mathrm{cg}$, and SN 2006X, which have a total extinction $A_{V}>1$ (see Section 4.3); but their $A_{V}$ due to the CSM extinction is lower than unity according to our models (see Section 5.2). Hence, ignoring multiple scattering is a reasonable approximation.

We assume that the observed flux is the sum of the light coming directly from the $\mathrm{SN}$ and the $\mathrm{SN}$ light scattered by the CSM, i.e., LEs. The direct flux contribution from the $\mathrm{SN}$ at a given epoch $t$ that is extincted and scattered by intervening dust without including the contribution from LEs can be written as

$$
f(t, \lambda)=f^{0}(t, \lambda) e^{-\left[\sigma_{s}(\lambda)+\sigma_{a}(\lambda)\right] N_{\text {tot }}}
$$

where $f^{0}$ is the intrinsic flux of the $\mathrm{SN}, \sigma_{s}(\lambda)$ and $\sigma_{a}(\lambda)$ are the cross sections of the dust particles for scattering and absorption of photons at wavelength $\lambda$, respectively. $N_{\text {tot }}$ is the total column density of dust between the $\mathrm{SN}$ and the observer and is equal to $N_{\text {ISM }}+N_{\text {CSM }}$. Then, the total observed flux adding the LE contribution can be expressed as

$$
\begin{aligned}
F(t, \lambda) & =f(t, \lambda)+\mathrm{LE} \\
& =f^{0}(t, \lambda) e^{-\sigma(\lambda) N_{\mathrm{tot}}}+S(t, \lambda) e^{-\sigma(\lambda) N_{\mathrm{ISM}}}
\end{aligned}
$$

where the first term is the intrinsic flux of the $\mathrm{SN}$ extincted by the total column density of dust (Equation (1)) and the second term is the contribution from LEs, $S(t, \lambda)$, extincted by ISM dust. $\sigma(\lambda)$ is the sum of the scattering and absorption cross sections. We can express $S(t, \lambda)$ as the sum of the light scattered by the CSM at different angles and epochs:

$$
\begin{aligned}
S(t, \lambda)= & \int f^{0}(\operatorname{tr}, \lambda) w(\lambda)\left(1-e^{-\sigma(\lambda) N_{\mathrm{CSM}}}\right) \\
& \times \Phi(\theta, \lambda) d \Omega \\
\operatorname{tr}=t- & \frac{D^{\prime}(\theta)-D}{c} \approx t-\frac{R(1-\cos (\theta))}{c}
\end{aligned}
$$

where $w(\lambda)\left(1-e^{-\sigma(\lambda) N_{\mathrm{CSM}}}\right)$ represents the fraction of light scattered by the CSM, which has a column density of $N_{\mathrm{CSM}} \cdot c$ is the speed of light, $w(\lambda)$ is the dust albedo and tr is a pseudo retarded time, i.e., the time at which a scattered pulse of light has to be emitted to reach the observer at the same time as a pulse emitted at time $t$ going straight to the observer. $D$ is the distance between the photosphere of the $\mathrm{SN}$ and the observer and $D^{\prime}(\theta)$ is the path length travelled by a photon being scattered by the CSM to the observer at an angle $\theta$ (see Figure 1).

$\Phi(\theta, \lambda)$ is the scattering phase function proposed in Henyey $\&$ Greenstein (1941):

$$
\Phi(\theta, \lambda)=\frac{1}{4 \pi} \frac{1-g(\lambda)^{2}}{\left(1+g(\lambda)^{2}-2 g(\lambda) \cos (\theta)\right)^{3 / 2}}
$$




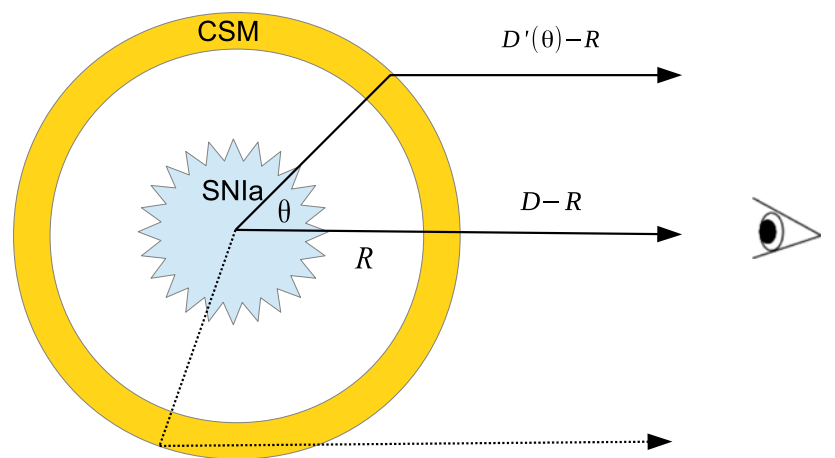

Figure 1. Sketch of the CSM model. Part of the light emitted by the SN is scattered by the CSM and redirected to the observer, arriving as a light echo delay.

where $g$ is the degree of forward scattering. When $g=1$ we have complete forward scattering and $g=0$ means isotropic scattering $(\Phi(\theta, \lambda)=1 /(4 \pi))$. We also define the parameter $f_{\text {CSM }} \equiv N_{\text {CSM }} / N_{\text {tot }}$ to simplify the notation, taking values between 0 and 1 . Finally, defining a delay parameter $\tau \equiv t-\operatorname{tr}$, an extinction factor $X(\lambda) \equiv e^{-\left(\sigma_{s}(\lambda)+\sigma_{a}(\lambda)\right) N_{\text {tot }}}$, and making a change of variable, using Equation (4), we obtain

$$
\begin{aligned}
S(t, \lambda)= & \frac{\omega(\lambda)\left(1-X^{f_{\mathrm{CSM}}}\right)}{\tau_{\max }} \\
& \times \int_{0}^{\tau_{\max }} f^{0}(t-\tau, \lambda) \Phi^{\prime}(\tau, \lambda) d \tau
\end{aligned}
$$

where $\tau_{\max }=2 R / c$ is the maximum delay for a light echo $(\theta=\pi)$ and $\Phi^{\prime}(\tau, \lambda)=4 \pi \Phi(\theta(\tau), \lambda)$. We performed this integral numerically using Simpson's $1 / 3$ integration rule and a time step of one day to simulate spectra and to fit this model to real data.

\section{LE MODEL PREDICTIONS}

To simulate spectra with different extinctions and LEs using Equation (6) we need to adopt a dust albedo, an extinction law, a phase function or $g(\lambda)$, and spectral templates with no extinction nor LEs at different epochs. For the extinction law we take the parameterization proposed in Fitzpatrick (1999). We use the albedo $w(\lambda)$ and the degree of forward scattering $g(\lambda)$ from the MW used in Goobar (2008), which accounts for the dust properties of the CSM. We construct unreddened spectral templates at different epochs from weighted bootstrapped averages of observed spectra of slow $B-V$ Lira law decliners (see Section 4.2), together with light-curve templates that we need since we normalize the spectra by their $V$-band flux (see Section 4.1). In Figure 2 we show the different scenarios for late-time (Lira law phase) model spectra when pure extinction and simulated LEs affect the SN emission.

We search for a way to distinguish if part of the dust found at maximum light is producing LEs. LE spectra are integrated spectra weighted by the light curve, and thus dominated by spectra around the peak (see Figure 2 with peak template spectrum and LE spectrum). LE spectra are blue and have very strong broad emission and absorption lines, with prominent peaks at $4000,4600,4900 \AA$ and minima at 4400 and $6200 \AA$.

When LE spectra are added to SN spectra: (1) the fact that the LE spectra are blue has a low-order effect on the observed
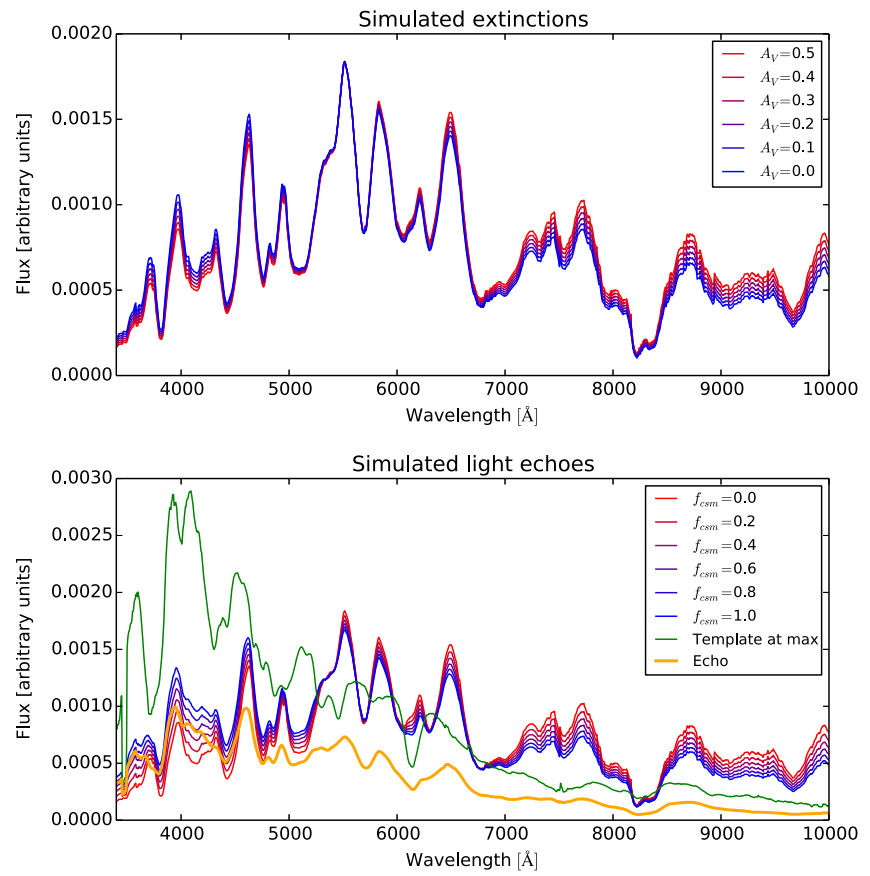

Figure 2. Upper panel: simulated spectra at 50 days past maximum light extincted by different amounts of dust $\left(A_{V}=0-0.5\right)$ with the same $R_{V}=3.1$ extinction law. All spectra have been normalized to the same $V$-band flux. Bottom panel: simulated spectra at 50 days past maximum light with the same reddening law, but also with LEs due to CSM. We fixed $A_{V}=0.5$ and varied the fractional amount of CSM $\left(f_{\mathrm{CSM}}\right)$ with a radius of $0.05 \mathrm{pc}$. The green line represents a typical maximum light spectrum, while in orange the LE spectrum is shown on an arbitrary scale. All models are normalized to the same $V$-band flux.

spectra by making the colors bluer, similar to less reddening and thus difficult to differentiate; (2) the strong broad lines add an additional modulation to the observed spectra that is very distinct to the effect of reddening, since it introduces differences on scales of a couple of hundred angstrom. By looking specifically at the wavelengths where the LE spectra have peaks or minima, it is possible to differentiate between the two scenarios. In Section 4 we compare these simulations to the observed spectra of SNe Ia.

In this simulation the main signature due to LEs is found near $4100 \AA$. This can be seen in Figure 3, where the shape of the spectrum gets considerably modified in the LE scenario (purple and blue lines), producing a characteristic signature. On the other hand, in the pure extinction scenario if the column density is reduced (black line), it produces just a smooth change in the spectrum compared to the same spectrum with extinction (reddest line). In particular, the shape of the feature near $4100 \AA$ will not be affected.

These features change with the distance $R$ between the CSM and the SN. Reducing the distance is analogous to making $\operatorname{tr}=t$ (see Equation (4)), obtaining the result of a pure extinction model where $S(t, \lambda)=\omega(\lambda)\left(1-X^{f_{\text {CSM }}}\right) f^{0}(t, \lambda)$. On the other hand, increasing $R$ makes the light echoes more diluted in time, reducing the ratio between the LE and the intrinsic flux $f$. Hence, there is an optimum $R$ near $0.05 \mathrm{pc}$ at which LEs can affect the colors and spectra during the Lira phase.

To further investigate the LE effects on spectra, we focus on the range between 3000 and $5000 \AA$, particularly on the absorption lines and their related measurable quantities such as the equivalent width (EW) and the slope of the continuum. We 


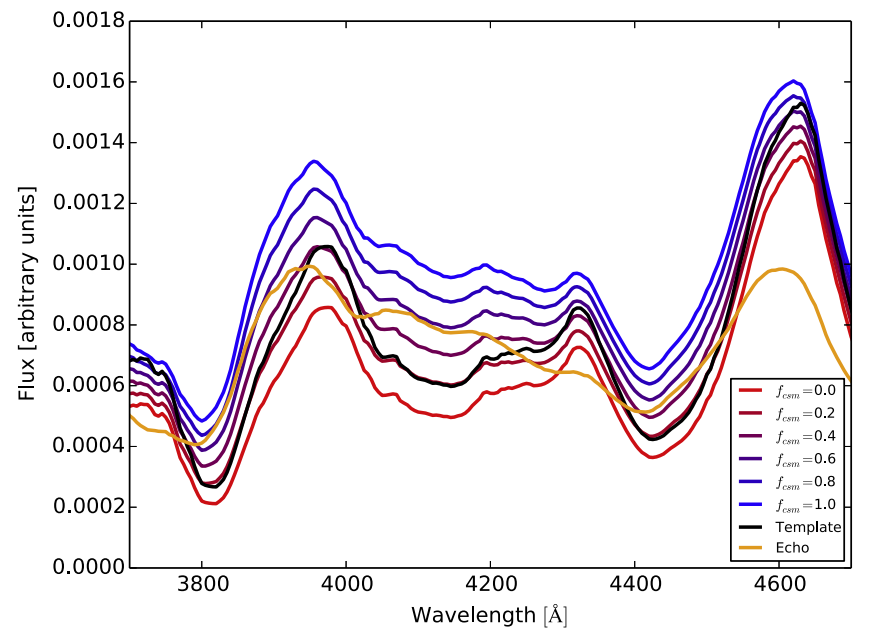

Figure 3. Simulated spectra at 50 days past maximum light with total extinction $A_{V}=0.5$ and $R_{V}=3.1$, and LEs due to CSM. The different lines represent different fractional amounts of CSM $\left(f_{\mathrm{CSM}}\right)$ with a radius of $0.05 \mathrm{pc}$ and a fixed total column density. The black line represents the original spectrum at 50 days past maximum light without extinction or LEs, while in orange the LE spectrum is shown on an arbitrary scale. All models are normalized to the $V$-band flux.

define five characteristic features, four of which are presented in Figure 4: the $\mathrm{Ca}$ II $\mathrm{H}$ and $\mathrm{K}$ complex between 3500 and $4100 \AA$, and two absorption features originating mainly from a blend of $\mathrm{Mg}$ II and $\mathrm{Fe}$ II at 3800-4400 $\AA$ ("line 1") and 4250-4800 $\AA$ ("line 2"); these two together form another larger feature at 3800-4800 $\AA$ ("line 3"), equivalent to the feature pW3 in Folatelli et al. (2013). Additionally, we use the line feature around the Si II absorption around 5800-6300 $\AA$ ("line 4")

For all LE and extinction models we measure pseudoequivalent widths $(\mathrm{pW})$, i.e., with a pseudo-continuum in a similar fashion to Garavini et al. (2007b), Bronder et al. (2008), and Folatelli et al. (2013). We use a semi-automatic algorithm that searches for the pseudo-continuum at specific regions defined in Table 1 . The algorithm also calculates the slope of the pseudo-continuum, which we find to be another good indicator of LEs. In Figure 5 we show the predicted evolution of the pWs of line 1 for a SN with pure extinction (green lines) and with LEs due to CSM (black lines). The pWs vary significantly during the Lira phase for LE models with different CSM fractions, $f_{\mathrm{CSM}}$, whereas they do not for different amounts of extinction. This effect is strongest at larger CSM distances of $0.05-0.25 \mathrm{pc}$. The difference between the scenario with extinction and with LEs is still clearer and less biased by the $\mathrm{SN}$ intrinsic $\mathrm{pWs}$ if we normalize by the $\mathrm{pW}$ at maximum light (hereafter $\mathrm{pW}$ ratio).

From the five $\mathrm{pW}$ ratios and respective slopes, we find that the best candidate to be a CSM indicator is line 1; the other lines show fewer differences in their evolution between different extinctions and CSM scenarios.

\section{COMPARISON WITH OBSERVED SPECTRA}

LEs are faint, and therefore they do not contribute significantly to the SN spectrum around maximum. Following this reasoning, we first analyze the reddening at maximum light, to determine the total extinction or $A_{V}$ due to CSM and/or ISM using standard extinction laws (see Section 4.3). Then,

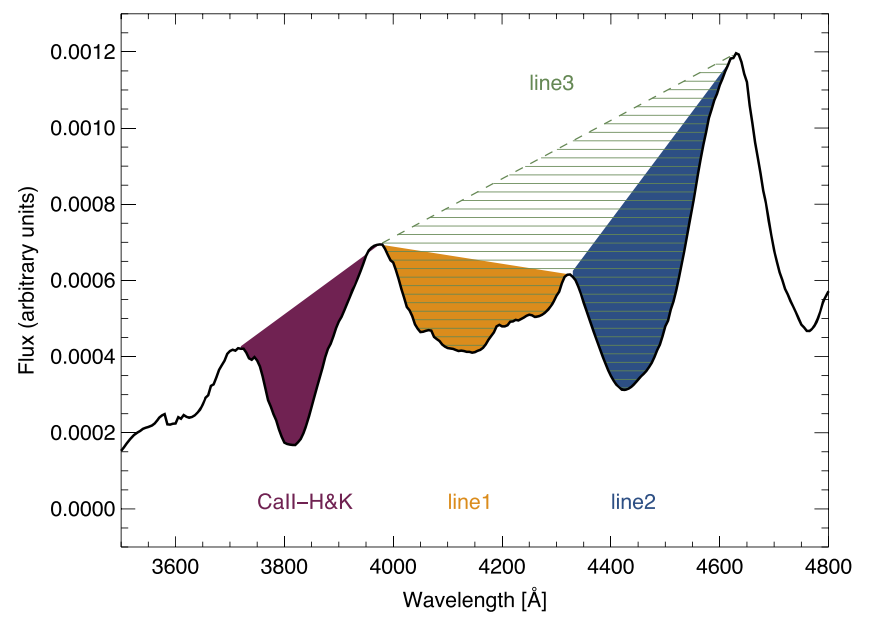

Figure 4. Features considered in the analysis as possible indicators of LE signatures: Ca II H and K complex at 3500-4100 А, "line 1" at 3800-4400 $\AA$, "line 2" at 4250-4800 $\AA$ and "line 3" at 3800-4800 $\AA$, the union of line 1 and line 2. Lines 2 and 3 come from a blend of $\mathrm{Mg}$ II and Fe II. The shaded regions indicate the $\mathrm{pW}$ for $\mathrm{Ca}$ II $\mathrm{H}$ and $\mathrm{K}$, lines 1 and 2 and the dotted line shows the pseudo-continuum of line 3 .

Table 1

Feature Definitions for Pseudo-equivalent Width and Pseudo-continuum

\begin{tabular}{lcc}
\hline \hline Feature & Blueward Limit Range $(\AA)$ & Redward Limit Range $(\AA)$ \\
\hline Ca II & $3500-3800$ & $3900-4100$ \\
Line 1 & $3800-4100$ & $4250-4400$ \\
Line 2 & $4250-4400$ & $4400-4800$ \\
Line 3 & $3800-4100$ & $4400-4800$ \\
Line 4 & $5800-6000$ & $6100-6300$ \\
\hline
\end{tabular}

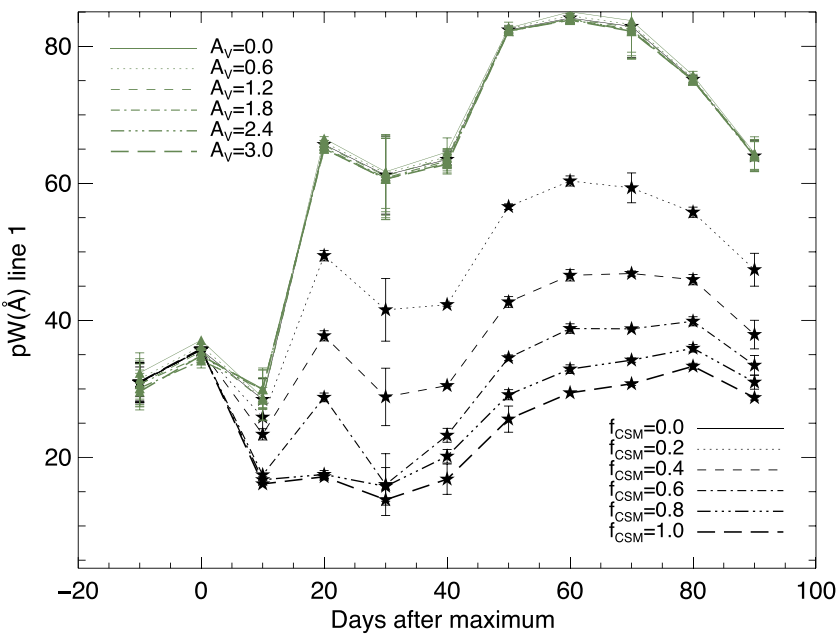

Figure 5. Evolution of the pseudo-equivalent width of the line 1 feature for simulated spectra. The green lines represent the evolution of line 1 with different amounts of extinction $\left(A_{V}\right)$, while the black lines are with different CSM amounts $\left(f_{\mathrm{CSM}}\right)$, with fixed radius of $0.05 \mathrm{pc}$ and a total column density or $A_{V}=1.0$.

analyzing the best fits to the spectrum at later epochs, we can discern if it is compatible with the extinction found at maximum light or if it is necessary to include LEs or, alternatively, reduce the amount of dust in the line of sight. 
Table 2

Nearby SNe Ia Spectra Used in this Analysis (Besides the Data from CSP, CfA and BSNIP) from the Online Supernova Spectrum Archive (SUSPECT)

\begin{tabular}{|c|c|}
\hline Name & Sources \\
\hline SN 2005hk & $\begin{array}{l}\text { Phillips et al. (2007), Blondin et al. (2012), Chornock } \\
\text { et al. (2006) }\end{array}$ \\
\hline SN 1999ac & Garavini et al. (2005) \\
\hline SN 1998aq & Branch et al. (2003) \\
\hline SN 2005cf & $\begin{array}{l}\text { Garavini et al. (2007a), Wang et al. (2009), Bufano et al. } \\
\text { (2009), Leonard (2007) }\end{array}$ \\
\hline SN 2003du & $\begin{array}{l}\text { Stanishev et al. (2007), Anupama et al. (2005), Gerardy \& } \\
\quad \text { Turatto (2005) }\end{array}$ \\
\hline SN 2005am & Leonard (2007) \\
\hline SN 2006X & $\begin{array}{l}\text { Yamanaka et al. (2009), Wang et al. (2008b), Sternberg et al. } \\
\text { (2011a) }\end{array}$ \\
\hline SN 1999aa & Garavini et al. (2004) \\
\hline SN 2002bo & Benetti et al. (2004) \\
\hline SN 2000cx & Li et al. (2001) \\
\hline SN 1994D & Patat et al. (1996), Gómez \& López (1998) \\
\hline SN 2003cg & Elias-Rosa et al. (2006) \\
\hline
\end{tabular}

\subsection{Data}

The spectra we use were taken from the Carnegie Supernova Program (CSP; Folatelli et al. 2013), public data of the Center for Astrophysics (CfA; Blondin et al. 2012b), the Berkeley Supernova Ia program (BSNIP; Silverman et al. 2013b), and The Online Supernova Spectrum Archive SUSPECT (see Table 2). We analyzed only the subset of SNe that were already classified as fast or slow Lira decliners during the Lira law phase in F13.

First, the spectra are corrected for MW extinction using the values from Schlafly \& Finkbeiner (2011) and de-redshifted to the rest frame. Then we smooth the spectra using a nonparametric fit with a velocity window of $1000 \mathrm{~km} \mathrm{~s}^{-1}$ and a wavelength regridding of $5 \AA$. We also compute the dispersion of each original spectrum with respect to its smoothed version to estimate the noise in our smoothed spectra. We normalize each smoothed spectrum to the same $V$-band flux by numerically convolving the spectrum with the filter transmission function (Bessell 1990), in order to put all spectra on the same scale and be able to compare the shape and features of the spectra instead of the absolute fluxes, which are difficult to calibrate precisely. We also adjust the shape of the spectra to match the observed colors interpolated to the given epoch (Hsiao et al. 2007), in order to have spectra consistent with the available photometry and with the previous work in F13.

We analyze the different SN spectra at maximum light and during the Lira law phase at five different epochs or time windows centered at 40, 50, 60, 70, and 80 days after maximum light with a width of 10 days. To have a single representative spectrum at every time window per $\mathrm{SN}$, we make weighted average spectra with the available spectra of each SN. For more details about these weighted averages refer to Appendix A.

\subsection{Template Spectra}

F13 showed that slow Lira decliners present weaker equivalent widths (EWs) of blended $\mathrm{Na}$ I D1 \& D2 narrow absorption lines, while fast Lira decliners have stronger EWs and redder colors independent of environmental factors. One possible interpretation of these results is that fast Lira decliners
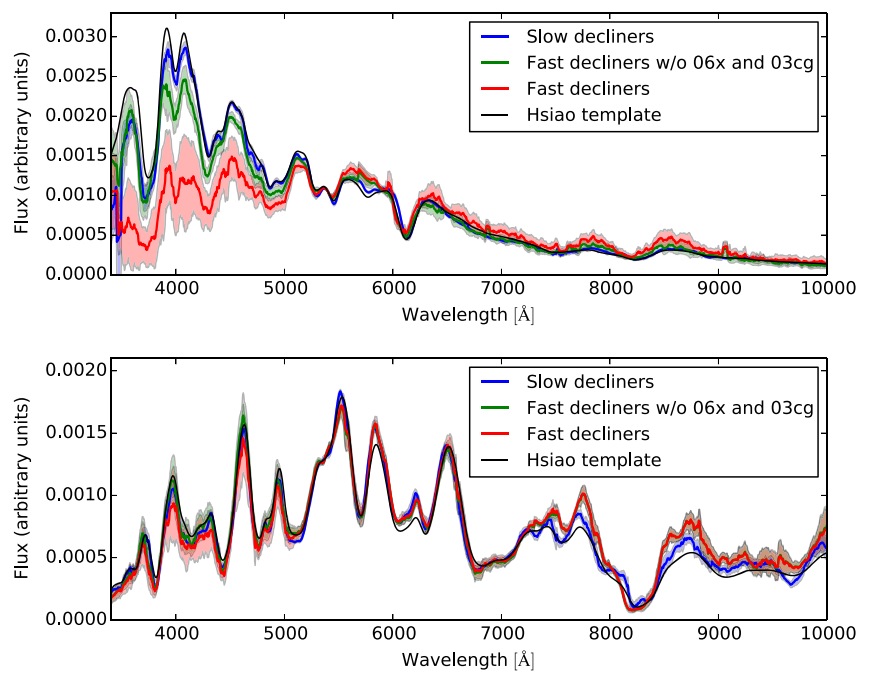

Figure 6. Bootstrap templates for slow and fast Lira decliner SNe at maximum light (upper panel) and 50.0 days after maximum light (lower panel), for a stretch of 0.98. In blue is the $B-V$ slow Lira decliner template, in red (green) is the $B-V$ fast Lira decliner template including (excluding) SN 2003cg and SN 2006X. The black line presents a template by Hsiao et al. (2007). The filled contours represent the one-standard-deviation regions based on the dispersion in the spectra.

have CSM that produces LEs or that they have nearby dust that is sublimated in time. In order to test these hypotheses and analyze the spectra of fast Lira decliners with our models, we compare them with some standard unreddened and nonevolving CSM spectral time series given in this case by the slow Lira decliners. For this, we construct different template spectra that cover the intrinsic SNe Ia variability at different epochs. Chotard et al. (2011) showed that most of the intrinsic variability of spectra in SNe Ia at 2.5 days after maximum light can be characterized with the EW of Si II $4131 \AA$ and $\mathrm{Ca}$ II $\mathrm{H}$ and $\mathrm{K}$. We follow that approach and use these two lines and a stretch parameterization to describe the shape of the light curve, defined as a factor that multiplies the time axis (Perlmutter et al. 1997; Goldhaber et al. 2001) to construct different templates. However, we cannot measure the EWs of the $\mathrm{Ca}$ and $\mathrm{Si}$ lines in all of our SNe Ia. Given than the EW of Si II 4131 is correlated with the light curve stretch parameter, we decide to use epoch and stretch as our main variables to construct templates accounting for the intrinsic variability in $\mathrm{SNe}$ Ia spectra.

To ensure that these average templates are not heavily biased by a few extreme $\mathrm{SNe}$, we perform a bootstrap simulation, i.e., we constructed 100 different templates using random sets from the original. For more details see Appendix B. In Figure 6 we compare the bootstrap templates for fast and slow Lira decliner spectra at maximum light and 50 days later, and one-standarddeviation $(\sigma)$ regions. At maximum light there is a clear difference between fast and slow Lira decliners near $4000 \AA$; the average difference is about $1.4 \sigma$ between 3400 and $10000 \AA$, and $0.8 \sigma$ if we do not consider SN 2006X and SN $2003 \mathrm{cg}$. Between 3400 and $5500 \AA$ the difference is about $2.3 \sigma$, and $1.4 \sigma$ if we do not consider SN 2006X and SN $2003 \mathrm{cg}$. But this difference after 50 days decreases to $0.8 \sigma$ between 3400 and $10000 \AA(0.9 \sigma$ without SN 2006X and SN $2003 \mathrm{cg})$ and $0.5 \sigma$ between 3400 and $5500 \AA$ ( $0.6 \sigma$ without SN 2006X and SN 2003cg). We also find that the dispersion among fast Lira decliners is larger than in our sample of slow 
Lira decliners. These results confirm what was found in F13, and we stress the fact that they are valid irrespective of warping the spectra to the observed colors. Finally, we point out that the template of the slow Lira decliners is quite similar to the template by Hsiao et al. (2007), suggesting that this is the group of more "normal" unreddened SNe Ia.

\subsection{Extinction Law at Maximum Light}

To find an extinction law for each fast Lira decliner at maximum light, we use as unreddened reference a slow Lira decliner template representing the same epoch and stretch, and the extinction law described in Fitzpatrick \& Massa (1990), adopting the mean values for the parameters found in Fitzpatrick (1999) and leaving the visual extinction $A_{V}$ as a free parameter.

We fix the total-to-selective extinction ratio $R_{V}$ at the standard MW value of 3.1. A discussion on this is presented in Section 6. For each fast Lira decliner average spectrum we fit $A_{V}$ and a normalization parameter $C_{1}$, which corrects the fact that our spectra are normalized to their $V$-band flux, minimizing a chi-square function (for more details see Appendix $C$ ).

To analyze global differences between the two subsets of $\mathrm{SNe}$ we fit the extinction law of Fitzpatrick (1999) to the bootstrap templates of fast Lira decliners using as reference the bootstrap templates of slow Lira decliners presented in Figure 12. We find at maximum light an extinction with $A_{V}=0.64 \pm 0.04$ and $R_{V}=3.0 \pm 0.2$ when we include the highly reddened SN $2006 \mathrm{X}$ and SN $2003 \mathrm{cg}$. When we exclude them we obtain $A_{V}=0.24 \pm 0.01$ and $R_{V}=3.3 \pm 0.1$. However, during the Lira law we obtain an extinction with $A_{V}=0.17 \pm 0.01$ and $R_{V}=3.3 \pm 0.1$ significantly lower than at maximum light and when we exclude these $\mathrm{SNe}$ we obtain $A_{V}=0.04 \pm 0.01, \quad R_{V}=3.6 \pm 0.1$. These results recover some of the results in F13: fast Lira decliners show a greater extinction at maximum light, which starts to decrease during the Lira law. Moreover, their $R_{V}$ slightly increases over time.

\subsection{LE Fit}

Using the model described in Section 2 plus the values for the dust albedo $w$ and the degree of forward scattering $g$, corresponding to dust with $R_{V}=3.1$, we fit the LE model to observed spectra of fast Lira decliners in the Lira phase. The parameters to fit are $f_{\mathrm{CSM}}$ and a normalization constant $C_{2}$ to correct for the fact that our spectra were normalized by their flux in the $V$-band.

The function to minimize for each average spectrum $i$ is

$$
\chi_{i}^{2}=\sum_{\lambda} \frac{\left(f^{i}(t, \lambda)-C_{2} F\left(t, f_{\mathrm{CSM}}, \lambda\right)\right)^{2}}{\delta f^{i}(t, \lambda)^{2}+\left(C_{2} \delta F\left(t, f_{\mathrm{CSM}}, \lambda\right)\right)^{2}}
$$

where

$$
F(t, \lambda)=f^{0}(t, \lambda) 10^{-0.4 A(\lambda)}+S(t, \lambda) 10^{-0.4 A(\lambda)\left(1-f_{\mathrm{CSM}}\right)} .
$$

To evaluate $S(t, \lambda)$ we use Equation (6) in a slightly different version because we do not have the intrinsic fluxes in our spectra as they were previously normalized (for more details, see Appendix D).

\section{RESULTS}

\subsection{Extinctions}

Out of 31 individual spectra from different fast Lira decliners at maximum light, minimizing the $\chi^{2}$ of Equation (19) we obtain $24 \mathrm{SNe}$ with positive $A_{V}$ values and 7 with unphysical negatives values. Those $\mathrm{SNe}$ are excluded from our sample for the LE analysis as they present less extinction than the template at maximum light, making the posterior fit of our LE model impossible. This may be caused by some $\mathrm{SNe}$ in the sample of slow Lira decliners having non-negligible host extinction. At maximum light the mean $A_{V}$ is 0.44 while excluding the highly reddened SNe, SN 2006X and SN 2003cg, it decreases to 0.27. But during the Lira law the amount of extinction starts to decrease to values near zero. In Table 3 the mean differences in $A_{V}$ per SN are presented with respect to the value found at maximum light, excluding those $\mathrm{SNe}$ with negative $A_{V}$ at that epoch. This result confirms that fast Lira decliners are more extincted at maximum light than slow Lira decliners, and during the Lira law the two groups become more similar, as shown in F13. This is also valid without the warping of the spectra to the observed colors. However, there are a few SNe that show a positive $\Delta A_{V}$. This could be produced by an artifact in our templates or a bad extinction law fit.

We find a small correlation between the $A_{V}$ values found at maximum light and the $B-V$ slope of the Lira law, with a Pearson's correlation coefficient of -0.5 (see Figure 7 ), which is consistent with the analysis done in F13.

\subsection{Light Echoes}

Once an $A_{V}$ per fast Lira decliner SN is found, we fit our LE model to the different fast Lira decliner spectra to search for spectral evidence of LEs. We look for the minimum of Equation (7) by varying $f_{\mathrm{CSM}}$ and $C_{2}$. We fix $R_{V}$ to 3.1 and the radius of the CSM shell to $0.05 \mathrm{pc}$ (more on this in Section 6).

We also fit the data with two other models to compare the goodness of fit of the LE model applied to the observations. The three models that we compare are:

1. The LE model in which we fit for $f_{\mathrm{CSM}}$ and $C_{2}$. It also includes ISM extinction to get a total extinction consistent with the amount of extinction found at maximum light.

2. A pure ISM model that just uses a late-time spectral template with the same extinction found at maximum light. In this scenario we just fit a normalization constant $C_{1}$.

3. A DS model consisting of a new pure extinction fit during the Lira law, with a lower $A_{V}$ value than the one found at maximum light.

Then we calculate the Bayesian information criterion (BIC) parameter in order to compare the goodness of the three models applied to the same data. The BIC parameter is obtained as

$$
\mathrm{BIC}=\chi^{2}+k \cdot \ln (n)
$$

where $k$ is the number of free parameters to fit in each model and $n$ is the number of data points or wavelengths with measured flux values. The best model is the one with the lowest BIC. It is a combination of $\chi^{2}$ plus a function that penalizes having too many free parameters overfitting the data. With the BIC parameter we judge whether the LE model is able to 
Table 3

Mean and Uncertainty of $\Delta A_{V}=A_{V}(t)-A_{V}(\max )$ for Fast Lira Decliner SNe at Different Epochs

\begin{tabular}{lcccc}
\hline \hline 40 days & 50 days & 60 days & 70 days & 80 days \\
\hline$-0.26 \pm 0.07$ & $-0.19 \pm 0.09$ & $-0.54 \pm 0.11$ & $-0.21 \pm 0.17$ & $-0.29 \pm 0.18$ \\
\hline
\end{tabular}

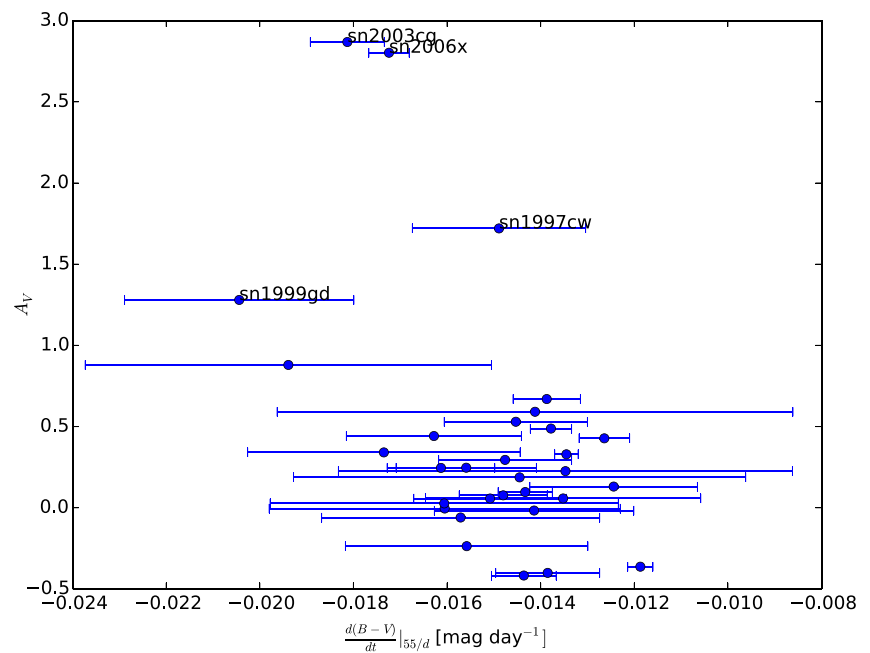

Figure 7. $A_{V}$ fit results at maximum light vs. $B-V$ slopes of the Lira law from F13.

explain the observed spectra better than a simple extinction or DS model. The results of the fits to the entire spectra and comparison between the three models are shown in Table 4.

According to our results, the LE model works better than using just the extinction law found at maximum light in $50 \%$ of the cases. But when we compare it to the DS model, the fraction of favorable cases drops to less than $20 \%$, giving larger weight to a scenario where $A_{V}$ simply decreases with time.

We also fit the three models to the spectra in particular wavelength ranges: blue region $(3000-5000 \AA)$; red region (6500-10000 $\AA$ ); signature I (3600-4800 $)$, and signature II (5900-6400 $\AA$ ). The results are shown in Table 5. The analysis in wavelength ranges is not favorable for the DS model, and the simple extinction found at maximum light works better than the LE model in most cases. However, this can be explained by the fact that when we look at particular regions of a spectrum a degeneracy appears between the normalization and the extinction, as there is not enough wavelength range to anchor the reddening, yielding a better BIC with the constant extinction model than with the DS model that has more parameters.

For each late-time SN spectrum, we derive the value for the CSM fraction $f_{\mathrm{CSM}}$. Multiplying this value with the $A_{V}$ found at maximum light, we can infer the fraction of the extinction due to CSM under the hypothesis of LEs. For the four SNe with $A_{V}>1$ at maximum light, we obtain that their $A_{V}$ 's due to CSM are lower than 0.5 , validating our single-scattering assumption.

In Figure 8 we show $f_{\mathrm{CSM}} \times A_{V}$ for different $\mathrm{SNe}$ at different epochs. Only epochs in which the LE model was the best according to its BIC value are plotted. If the CSM were not disturbed by the SN we would expect $f_{\mathrm{CSM}} \times A_{V}$ to stay almost constant for each SN. Unfortunately, only one object, SN 2003W, has more than one late-time spectrum consistent with LEs to allow us to perform this test. The three resulting
Table 4

Comparison of ISM, LE, and DS Models with Overall Spectra

\begin{tabular}{lccccc}
\hline \hline Comparison & 40 days & 50 days & 60 days & 70 days & 80 days \\
\hline Number of SNe & 16 & 11 & 8 & 10 & 7 \\
LE versus ISM & 0.50 & 0.36 & 0.63 & 0.5 & 0.57 \\
LE versus ISM & 0.19 & 0.18 & 0.13 & 0.2 & 0.14 \\
$\quad$ and DS & & & & & 0.43 \\
$\begin{array}{l}\text { DS versus ISM } \\
\text { and Echo }\end{array}$ & 0.44 & $0.27^{\mathrm{a}}$ & 0.63 & 0.3 & 0.43 \\
\hline
\end{tabular}

${ }^{\text {a }}$ The numbers show the fraction of fast Lira decliner SNe with positive $A_{V}$ at maximum light that have a BIC value which favors the LE or DS models vs. the other models at different time windows.

$f_{\text {CSM }} \times A_{V}$ values range between 0.03 and 0.23 , which we consider a satisfactory agreement considering the simplifications of the LE model.

The number of SNe for which the LE model has a favorable BIC in comparison with the ISM and DS models is small (seven SNe), and just SN 2003W appears in more than one epoch. Even in such cases, a visual inspection of the spectra does not reveal the signatures expected from LEs. This poses serious questions for the LE scenario.

\subsection{Line Comparison}

Another way to test our LE model is to use the line diagnostics presented in Section 3. For this we calculate for all our spectra the $\mathrm{pW}$ and pseudo-continuum slope for the four diagnostic lines previously defined, in the same way as we measure them in the simulated spectra. We obtain these features at all available epochs and also normalize the $\mathrm{pW}$ curves by their values at maximum light in order to study their evolution. Finally we compare the results for the slow and fast Lira decliners with the simulated spectra. Apart from some slight differences between the two samples, we find that in general the populations are consistent within the errors. Both groups of SNe Ia show a similar trend and dispersion in their evolution. As an example, Figure 9 shows the distribution of $\mathrm{pW}$ at 55 days after maximum light for the two SN samples. In the context of the LE model, we would expect a difference in both the dispersion of pWs and their mean value, neither of which is observed.

To measure possible statistical differences we perform a Kolmogorov-Smirnov (KS) test. We find that for line 1 the two populations, slow and fast Lira decliners, have a $99 \%$ probability of being drawn from the same distribution. We check all of the other lines for which we obtain low KS values, yet we do not find any hint of LE signatures among fast Lira decliners according to our predictions (KS values between 0.2 and 0.99). The $\mathrm{pW}$ distributions are consistent for both populations during the Lira phase. However, some pseudocontinuum slopes show very low KS values (lower than 0.01). This difference in both SN samples can be explained by the greater color dispersion of fast Lira decliners, which also tend to be redder at maximum light, as shown in F13. As a matter of 
Table 5

Comparison of ISM, LE, and DS Models With Specific Spectral Regions

\begin{tabular}{|c|c|c|c|c|c|}
\hline Comparison & $3900-10000 \AA$ & $3000-6500 \AA$ & $6500-10000 \AA$ & $3600-4800 \AA$ & 5900-6400 ̊ \\
\hline LE versus ISM & 0.36 & 0.45 & 0.45 & 0.27 & 0.18 \\
\hline LE versus ISM and DS & 0.18 & $0.36^{\mathrm{a}}$ & 0.45 & 0.27 & 0.18 \\
\hline DS versus LE and ISM & 0.27 & 0.18 & 0.0 & 0.0 & 0.0 \\
\hline
\end{tabular}

${ }^{\text {a }}$ The numbers show the fraction of fast Lira decliner SNe with positive $A_{V}$ at maximum light that have a BIC value which favors the LE or DS models vs. the other models at 50 days after maximum light for different wavelength regions.

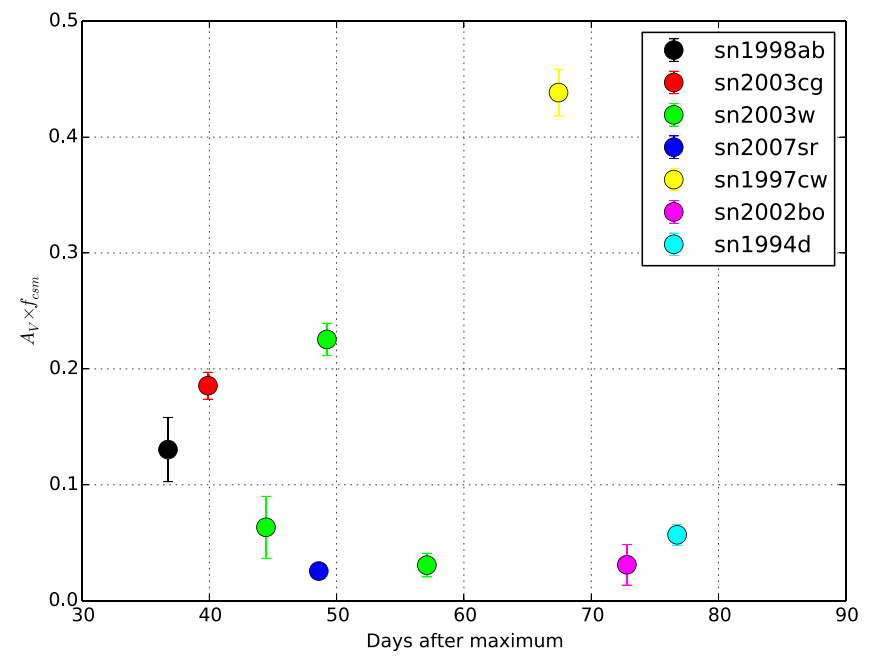

Figure 8. $A_{V} \times f_{\mathrm{CSM}}$ vs. time since maximum light. Only cases in which the LE model works better than the pure extinction and DS models are shown. The error bars represent errors of three standard deviations on the parameters that minimize the $\chi^{2}$ function under our LE model assumption.

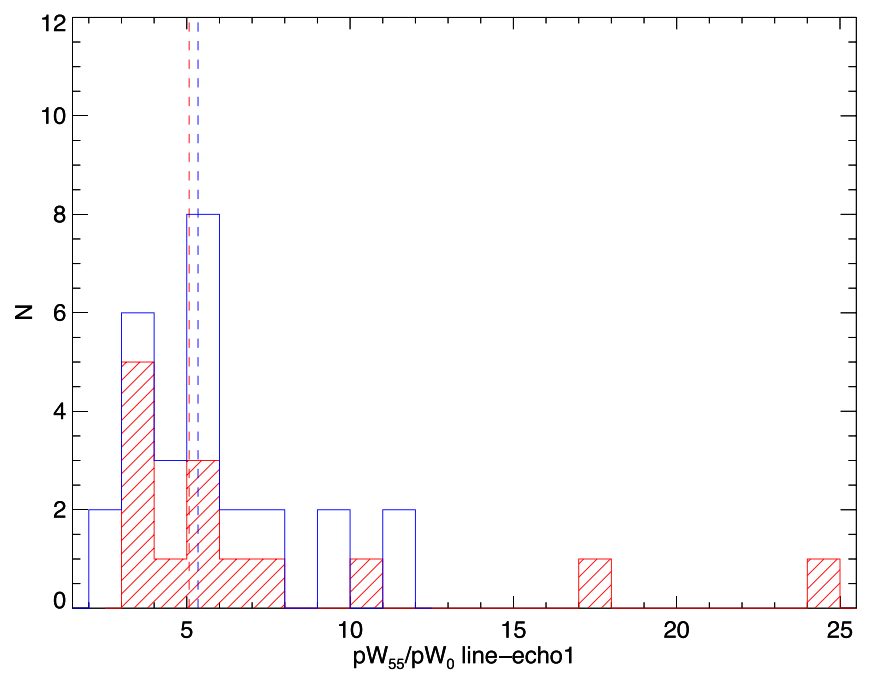

Figure 9. Histograms of the ratio of pseudo-equivalent width at maximum light and at 55 days past maximum light for the diagnostic echo line 1 for the sample of slow (blue) and fast (red) Lira decliners. Vertical dashed lines show the median of the population: $5.4 \pm 1.70$ for slow and $5.7 \pm 1.57$ for fast Lira decliners. The KS test for these distributions gives a probability of $99 \%$ of their being drawn from the same distributions.

fact, the trend goes in the opposite direction to what is predicted by our LE models.

Although we do not find a correlation between the evolution of lines and fast Lira decliners, our CSM model predictions, especially for line 1 , can be important to diagnose the presence of LEs in other samples of SNe Ia.

\section{DISCUSSION}

\subsection{Extinction Laws and $\mathrm{R}_{\mathrm{V}}$}

To calculate the extinction at maximum, we also use other reddening laws, such as Cardelli et al. (1989) with the inclusion of O'Donnell (1994), obtaining similar results to Fitzpatrick (1999) when keeping $R_{V}$ fixed. We also explore the reddening law proposed in Goobar (2008), but we discard it for two reasons: the reddening law becomes degenerate with our normalization constant $C_{1}$ and also because it accounts for the observed reddening in the context of CSM without considering the evolution of the radiated spectrum. In contrast, we aim to find an intrinsic reddening law and the time evolution of the spectrum caused by LEs.

To be consistent in our predictions and in our fits we used extinction laws with a fixed $R_{V}$ of 3.1 as we use standard MW values for the albedo and phase function of interstellar dust. Therefore our analysis is restricted to a statistical point of view rather than the study of particular cases. The $R_{V}$ value can vary depending on the properties of the dust (e.g., grain size distribution and composition) and seems to be different from the MW in the line of sight toward some SNe Ia (Mandel et al. 2011; Burns et al. 2014). It is not very clear what range of values are consistent with circumstellar dust surrounding a SN. It is very important to differentiate in analysis of extinction laws between the intrinsic $R_{V}$, which comes from the dust properties, and the observed $R_{V}$ when a pure extinction law is assumed, omitting more complex interactions. A future improvement in our model is to compute and use the specific opacities, albedo and phase function given any dust grain size distribution and composition.

\subsection{Light Echo Models}

We have explored the possibility of detecting LEs due to CSM in SNe Ia spectra. Our results show that LEs are not a global phenomenon on fast Lira decliner SNe during the Lira law phase. Even though we find that for $\sim 50 \%$ of the spectra the LE model works better than the extinction law derived at maximum light, the number of favorable cases drops to values near $15 \%$ when we compare them with the DS models.

We also fit the LE model using a CSM radius $R$ of 0.01 and $0.25 \mathrm{pc}$, instead of $0.05 \mathrm{pc}$. With the smallest $R$ we obtain a lower fraction of favorable cases for the LE model compared to the original results with $R=0.05 \mathrm{pc}$, even lower than $50 \%$ when the LE model is compared with just the pure extinction model (ISM). On the other hand, when we fix $R=0.25$ we recover almost the same results as the original CSM scenario. Therefore, if CSM is present, larger radii of $0.05-0.25 \mathrm{pc}$ are favored. In principle, it is possible to fit at the same time $R$, $f_{\mathrm{CSM}}$, and $C_{2}$, but this is computationally expensive and could overfit the data. 
Our CSM model consists of an isotropic spherical shell in the limit of negligible thickness. We did not consider multiple scattering, which in optically thin scenarios is negligible. Nevertheless, we know that multiple scattering could become important at optical wavelengths when the optical depth is larger than 1, i.e., $A_{V} \gtrsim 1$. Therefore we are unable to analyze $\mathrm{SNe}$ with an expected $A_{V}$ due to CSM larger than 1, but according to our results none of the $\mathrm{SNe}$ in our sample presented an $A_{V}$ due to CSM larger than 1 (including SN $2003 \mathrm{cg}$ and SN 2006x). A model that includes multiple scattering is necessary to predict the effect on the light curves and spectra when an optically thick CSM is present (Patat 2005; Amanullah \& Goobar 2011).

The CSM geometry may probably be different from a spherical shell, e.g., non-isotropic disk or ring geometries formed from a planetary nebula have been proposed recently to model time-variable Na I D absorption (Soker 2014). The predicted LE signatures might vary depending on the CSM geometry and orientation to the observer.

For an optically thin shell, a rough estimate of the total dust mass in our models can be obtained for a given CSM radius, an $A_{V}$, and a typical ISM dust opacity:

$$
\begin{aligned}
M_{d}= & 6.4 \times 10^{-5}\left(\frac{R}{0.01 \mathrm{pc}}\right)^{2}\left(\frac{A_{V}}{0.1}\right) \\
& \times\left(\frac{\kappa_{V}}{8.55 \times 10^{3} \mathrm{~cm}^{2} \mathrm{~g}^{-1}}\right)^{-1} M_{\odot}
\end{aligned}
$$

This is a very high mass in the form of dust for a CSM. However, we are assuming a spherical shell and a specific opacity corresponding to interstellar extinction in the MW. If we consider a different geometry for the CSM or a larger specific opacity, i.e., smaller grains, the inferred mass will vary by orders of magnitude.

\subsection{LE Effects on the Light Curves}

Using Monte Carlo simulations, Amanullah \& Goobar (2011) found that a different radius or $E(B-V)$ for the CSM could affect the $B-V$ evolution during the Lira law phase in different ways. In order to test the hypothesis made in F13 that LEs could increase the $B-V$ decline rate during the Lira law phase, we investigate whether our CSM model affects the behavior of the light curve during the Lira law phase. We find that LEs do the opposite: they tend to smooth the color evolution. This goes in the opposite direction to our goal of finding LEs in the spectra of fast Lira decliners and favors the DS model.

In Figure 10 we present these simulated light curves. As expected, in the pure extinction scenario the $B$ and $V$ light curves (red line) are just uniformly shifted downward with respect to the original light curve without extinction (black line). On the other hand, the presence of LEs due to CSM modifies the shape of these curves, increasing the brightness in $B$ and $V$ at later epochs as blue light from maximum light is reaching the observer. However, the slope of the $B-V$ evolution actually becomes shallower during the Lira law, see Table 6 . This can be explained qualitatively because our LE model adds light emitted at previous epochs to the intrinsic emitted light, including late-time emission with small time delays. These contributions make the observed light curve evolve slowly.
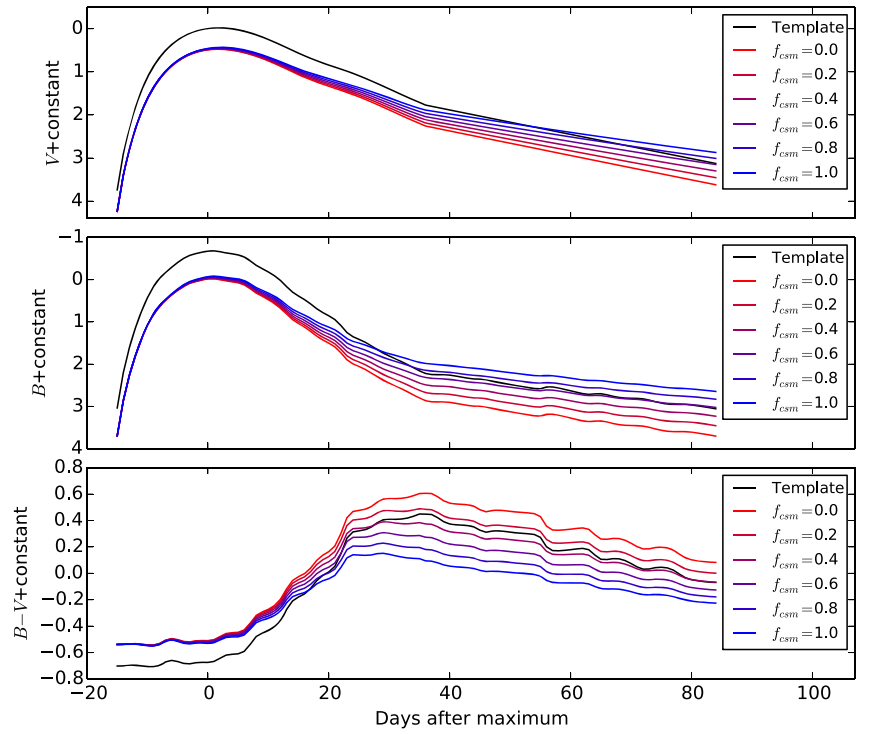

Figure 10. Simulated light curves using our LE model. In black is the original light curve without extinction. The reddest curve represents a light curve with pure extinction $\left(A_{V}=0.5\right.$ and $\left.R_{V}=3.1\right)$ and the rest are scenarios with different fractions of CSM.

Table 6

$B-V$ Slope During the Lira Law Phase from Simulated Light Curves

\begin{tabular}{lc}
\hline \hline$f_{\text {CSM }}$ & Slope \pm Error $($ mag/day $)$ \\
\hline 0.0 & $-0.010 \pm 0.002$ \\
0.2 & $-0.008 \pm 0.001$ \\
0.4 & $-0.008 \pm 0.001$ \\
0.6 & $-0.007 \pm 0.001$ \\
0.8 & $-0.004 \pm 0.001$ \\
1.0 & $-0.005 \pm 0.001$ \\
\hline
\end{tabular}

The wiggles in the $B$ and $B-V$ light curves in Figure 10 are not real. They are caused by the way we compute the $B$ magnitudes from the template spectra, which depend on the available SN spectra at each time window. On the other hand, the $V$ magnitudes match the observed photometry by construction. Despite this, the general shape of the light curves is clear.

\subsection{DS and its Effects on the Light Curve}

A decreasing extinction or opacity could occur if the CSM dust that was extincted at maximum light got sublimated by an increment of temperature due to the SN radiation, reducing the total opacity in the line of sight. This sublimation could also change the observed $R_{V}$ as it might change the grain size distribution and composition, explaining the evolution found in F13. If this happens during the Lira law phase, it will be reflected in a steeper $B-V$ slope. To test this hypothesis we simulated light curves with a decreasing $A_{V}$ and variable $R_{V}$. Figure 11 presents these three different scenarios. We found that a decreasing $A_{V}$ or increasing $R_{V}$ can make the $B-V$ evolution become steeper.

A smaller $R_{V}$ makes the extinction law more sensitive to the blue than the red, which is expected if the grain size distribution favors small sizes. An increasing $R_{V}$ could be produced if the smaller grains disappear as sublimation occurs. Another possibility is that the intrinsic CSM $R_{V}$ was lower than the ISM $R_{V}$, therefore as the circumstellar dust is being sublimated, the total $R_{V}$ increases, reaching values similar to 

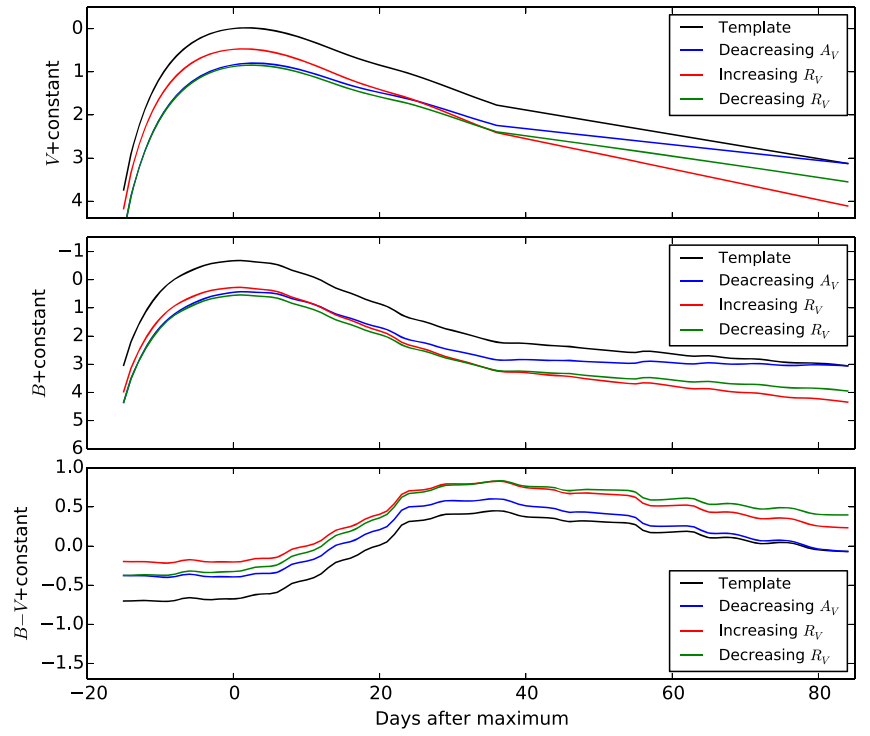

Figure 11. Simulated light curves using extinction laws that vary over time. In black is the original light curve without extinction. The blue curve is a model with $A_{V}$ decreasing from 0.5 to 0.0 and $R_{V}=3.1$ held constant. The red curve represents a light curve with $A_{V}=0.5$ and $R_{V}$ evolving from 2.0 to 3.1 . The green curve represents a light curve with $A_{V}=0.5$ and $R_{V}$ evolving from 3.1 to 2.0 .

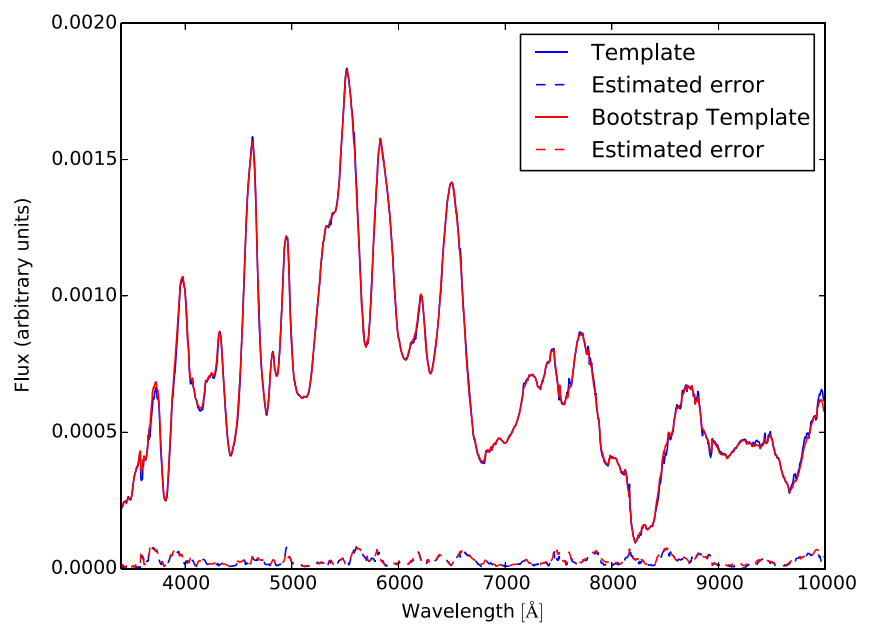

Figure 12. Template spectrum of a slow Lira decliner SN using weighted averages vs. using the bootstrap technique at 50 days after maximum light and using a stretch of 0.98 .

the interstellar $R_{V}$. This hypothesis is also consistent with the mean $\Delta A_{V}$ found at different epochs for fast Lira decliners.

Within this context, it is important to note that recently Goobar et al. (2015) showed that SN 2014J, an extincted red SN Ia with low $R_{V}$ (Foley et al. 2014) and with strong narrow absorption features (Welty et al. 2014), has possible signatures of cooling from shocked material from nearby CSM of dimensions larger than $1 R_{\odot}$. But the scales of this CSM are smaller than the ones considered in this paper.

Another possibility is that the radiation pressure (RP) of the $\mathrm{SN}$ is blowing away the CSM dust particles. If the CSM radius is increased, the observed extinction will decrease as the column density decreases, also producing blueshifted absorption lines. A rough estimation of the timescales on which the RP could produce an observable change in the CSM extinction can be calculated as

$$
\begin{gathered}
\tau_{\mathrm{RP}}=\frac{R_{\mathrm{CSM}}}{\Delta v} \\
\tau_{\mathrm{RP}}=\frac{m_{d} R_{\mathrm{CSM}} c}{f_{\mathrm{SN}} \sigma_{a} \Delta t}
\end{gathered}
$$

where $R$ is CSM radius and $\Delta v$ is a characteristic velocity of the dust grains after they absorb linear momentum from the SN incident radiation flux $f_{\mathrm{SN}}$ that can be calculated using the well known relation between the RP and flux of an electromagnetic wave. We consider spherical dust particles of radius $a$, internal density of $1 \mathrm{~g} \mathrm{~cm}^{-3}$, mass $m_{d}$, and with a cross section $\sigma_{a}$ calculated using the Mie theory. Assuming a typical SN Ia luminosity and a time range $\Delta t=25$ days, centered at maximum light, in which the RP injects momentum to the dust particles, we can obtain an estimate of the timescale on which this effect could be observed:

$$
\tau_{\mathrm{RP}}=1.4 \times 10^{2}\left(\frac{a}{1 \mu \mathrm{m}}\right)\left(\frac{R}{0.01 \mathrm{pc}}\right) \text { days } .
$$

Thus, if the RP is the cause of the decreasing extinction at $\tau_{\mathrm{RP}} \sim 80$ days past explosion (Lira law), the CSM dust particles should be smaller than $1 \mu \mathrm{m}$ or be at distances of $\sim 10^{3}$ AU. At these distances we expect that the sublimation timescales of these smaller grains are much shorter than those calculated above.

Therefore, even if DS timescales are too long to account for the changes in extinction and $R_{V}$, during the Lira law phase, we expect that the CSM expansion due to RP should increase the observed $R_{V}$ and decrease the extinction, as smaller grains are blown away.

An alternative scenario to the CSM sublimation and RP that could explain the decrease in extinction is the transverse expansion of the ejecta in an inhomogeneous ISM. This possibility is explored in F13 where they conclude that this scenario could explain the change in the average column density as the photospheric radius increases, but it does not explain the change in the observed $R_{V}$ over time.

\section{CONCLUSIONS}

A CSM model producing LEs has been developed. It is simple enough to be computed quickly and to be used in our fitting routines, but with enough complexity to account for the albedo of the dust and the scattering phase function. Our model predicts two spectral signatures produced by LEs at 4100 and $6200 \AA$ that can help us discriminate between a pure extinction and extinction+LEs scenario. These features appear within small wavelength ranges as opposed to overall color changes that can also be produced by reddening. The evolution of these signatures is another tracer of the presence of CSM producing LEs.

We compare our models with observed SN spectra. We find that LEs from CSM at $0.01-0.25$ pc are not a global phenomenon in fast Lira decliner SNe when they are compared to slow Lira decliners. ISM or CSM dust being sublimated (DS) at later epochs explains the observed spectra better when the models are fitted using the overall spectrum. Additionally, we find no evidence for LEs based on the narrow spectral diagnostics predicted by our model. 
We explore the effects on the light curve of circumstellar dust being sublimated or blown away by RP, finding that both scenarios could produce a faster $B-V$ decline during the Lira law and a change in $R_{V}$, although a more rigorous physical modeling is needed to explore these possibilities.

We laid out several ways to improve our models: adding a $R_{V}$ as a free parameter in our fits; using different CSM geometries to test our predictions; and a Monte Carlo radiative transfer simulation to see if the LE signatures remain in the multiple scattering scenario. The analysis can also be improved using a larger sample of $\mathrm{SNe}$, particularly those highly extincted and with possible CSM characteristics.

We thank the referee for providing constructive comments and help in improving the contents of this paper. We also thank K. Maeda for useful discussion. S.M. would like to thank CONICYT-PCHA/MagisterNacional/2014-folio 22140628. S.G. thanks CONICYT through FONDECYT grant 3130680 for its support. F.F. thanks CONICYT through FONDECYT grant 11130228 for its support. S.M., S.G., F.F., and M.H. acknowledge support provided by the Ministry of Economy, Development, and Tourism's Millennium Science Initiative through grant IC120009, awarded to The Millennium Institute of Astrophysics, MAS. E.Y.H. acknowledges the generous support provided by the Danish Agency for Science and Technology and Innovation through a Sapere Aude Level 2 grant.

\section{APPENDIX A \\ AVERAGE SPECTRA PER SN PER TIME RANGE}

For the $j$ th spectrum of the $i$ th $\mathrm{SN}$ we defined the following weights:

$$
w_{j}^{i}=e^{-\left(t_{j}-t_{0}\right)^{2} / 2 \sigma_{t}^{2}}
$$

where $t_{0}$ is the epoch that we want to represent and $\sigma_{t}=5.0$ days is half of the time window. The average spectrum for a particular $\mathrm{SN}$ at the given epoch $t_{0}$ was then calculated according to

$$
f^{i}(\lambda)=\frac{\sum_{j} w_{j}^{i} f_{j}^{i}(\lambda) / \delta f_{j}^{i}(\lambda)^{2}}{\sum_{j} w_{j}^{i} / \delta f_{j}^{i}(\lambda)^{2}}
$$

where $f_{j}^{i}(\lambda)$ and $\delta f_{j}^{i}(\lambda)$ are the normalized flux of the $j$ th spectrum and its error. The sum goes over all the available spectra of the $i$ th $\mathrm{SN}$ with measured fluxes in the given time range. We also computed an error and a representative epoch for each average spectrum in a similar way.

\section{APPENDIX B TEMPLATE SPECTRA}

To construct template spectra we define two weight factors for time and stretch of the $i$ th average spectrum of each slow Lira decliner SN:

$$
\begin{gathered}
w_{t}^{i}=e^{-\left(t_{i}-t_{0}\right)^{2} / 2 \sigma_{t}^{2}} \\
w_{s}^{i}=e^{-\left(s_{i}-s_{0}\right)^{2} / 2 \sigma_{s}^{2}}
\end{gathered}
$$

We chose $\sigma_{t}=1.5$ days and $\sigma_{s}=0.11$ in order to reproduce a specific epoch and stretch. We do not have strong arguments to choose particular values, thus we use the standard deviation of

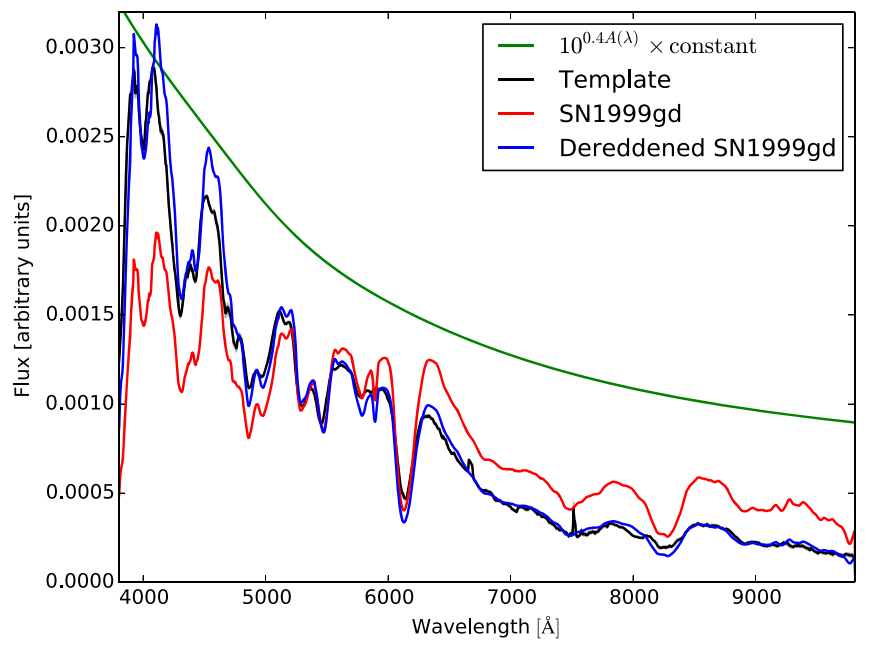

Figure 13. Extinction law fit for $\mathrm{SN} 1999 \mathrm{gd}$ at maximum light using the Fitzpatrick (1999) extinction law. The best fit with $R_{V}=3.1$ yields $A_{V}=1.280 \pm 0.003$. In red we present the observed spectrum of $\mathrm{SN}$ $1999 \mathrm{gd}$ at maximum light, in black a constructed template representing the same epoch and stretch. The blue line is the dereddened spectrum and the green line is the extinction factor $e^{-\tau \lambda}$ or $10^{-0.4 A \lambda}$ shape.

stretches in our sample for $\sigma_{s}$ and a $\sigma_{t}$ value smaller than our time windows but large enough to reproduce smooth light curves. Defining $\alpha_{i}=w_{t}^{i} \times w_{s}^{i}$, the template spectrum with a certain epoch $t_{0}$ and stretch $s_{0}$ is

$$
F(\lambda)=\frac{\sum_{i} \alpha_{i} f_{i}(\lambda) / \delta f_{i}(\lambda)^{2}}{\sum_{i} \alpha_{i} / \delta f_{i}(\lambda)^{2}}
$$

where the sum goes over all the available, already averaged spectra from slow Lira decliners with measured fluxes at wavelength $\lambda$. Finally we normalized the template spectrum by its flux in the $V$-band.

To ensure that these average templates are not heavily biased by a few extreme $\mathrm{SNe}$, we performed a bootstrap simulation. Then computing the mean template and the dispersion around it, we obtained a "bootstrap template" and its error. In Figure 12 we compare a template constructed using weighted averages and a template using the bootstrap simulation. In both cases we used the whole sample of slow Lira decliners. There are slight differences between the two spectra, but these are not significant and the estimated errors are very similar.

\section{APPENDIX C EXTINCTION LAW FIT}

To fit an extinction law we minimize a chi-square function for each average spectrum $i$ :

$$
\chi_{i}^{2}=\sum_{\lambda} \frac{\left(f^{i}(\lambda)-f^{0}(\lambda) 10^{-0.4 A(\lambda)} / C_{1}\right)^{2}}{\delta f^{i}(\lambda)^{2}+\left(\frac{f^{i}(\lambda)}{f^{0}(\lambda)} \delta f^{0}(\lambda)\right)^{2}}
$$

where $f^{i}(\lambda)$ is the normalized flux of the $i$ th fast Lira decliner $\mathrm{SN}$ at maximum light and $f^{0}(\lambda)$ is an unreddened template representing the same intrinsic flux. In Figure 13 we present a particular extinction law fit for SN 1999gd at maximum light. 
We found that $A_{V}=1.280 \pm 0.003$ best represents the extinction using our template spectrum.

\section{APPENDIX D \\ LIGHT ECHO FIT}

To fit our light echo model we introduce a new factor $N$ to force the spectra to evolve consistently with the light curve in the $V$-band. Hence we calculate $S(t, \lambda)$ as

$$
\begin{aligned}
S(t)= & \frac{w\left(1-10^{-0.4 A f_{\mathrm{CSM}}}\right)}{\tau_{\max }} \\
& \times \int_{0}^{\tau_{\max }} N(t, \tau) f^{0}(t-\tau) \Phi^{\prime}(\tau) d \tau \\
& N(t, \tau)=10^{-0.4(V(t-\tau)-V(t))}
\end{aligned}
$$

where we omitted the wavelength dependence of $S, f^{0}, \omega, \Phi^{\prime}$, and $A$ (the extinction law found at maximum light). $f^{0}(t)$ is a template spectrum representing the same intrinsic flux at time $t$. We multiply each spectrum by $N(t, \mathrm{tr})$, which is the $V$ light curve normalized in $t$, to correct for the fact that all our templates are normalized by their flux in the $V$-band. This factor is computed using weighted averages of the $V$ magnitudes of slow Lira decliners considering epoch and stretch as we did for the template spectra. The $V$ magnitude data were taken from SiFTO fits to the data (Conley et al. 2008).

\section{REFERENCES}

Amanullah, R., \& Goobar, A. 2011, ApJ, 735, 20

Anupama, G. C., Sahu, D. K., \& Jose, J. 2005, A\&A, 429, 667

Benetti, S., Meikle, P., Stehle, M., et al. 2004, MNRAS, 348, 261

Bessell, M. S. 1990, PASP, 102, 1181

Bianco, F. B., Howell, D. A., Sullivan, M., et al. 2011, ApJ, 741, 20

Blondin, S., Dessart, L., Hillier, D. J., \& Khokhlov, A. M. 2013, MNRAS, 429, 2127

Blondin, S., Prieto, J. L., Patat, F., et al. 2009, ApJ, 693, 207

Bloom, J. S., Kasen, D., Shen, K. J., et al. 2012, ApJ, 744, L17

Branch, D., Garnavich, P., Matheson, T., et al. 2003, AJ, 126, 1489

Bronder, T. J., Hook, I. M., Astier, P., et al. 2008, A\&A, 477, 717

Bufano, F., Immler, S., Turatto, M., et al. 2009, ApJ, 700, 1456

Burns, C. R., Stritzinger, M., Phillips, M. M., et al. 2014, ApJ, 789, 32

Cappellaro, E., Patat, F., Mazzali, P. A., et al. 2001, ApJ, 549, L215

Cardelli, J. A., Clayton, G. C., \& Mathis, J. S. 1989, ApJ, 345, 245

Chevalier, R. A. 1986, ApJ, 308, 225

Chomiuk, L., Soderberg, A. M., Moe, M., et al. 2012, ApJ, 750, 164

Chornock, R., Filippenko, A. V., Branch, D., et al. 2006, PASP, 118, 722

Chotard, N., Gangler, E., Aldering, G., et al. 2011, A\&A, 529, L4

Conley, A., Carlberg, R. G., Guy, J., et al. 2007, ApJ, 664, L13

Conley, A., et al. 2008, ApJ, 681, 482

Crotts, A. 2014, e-print (arXiv:1409.8671)

di Stefano, R. 2010, ApJ, 712, 728

di Stefano, R., Voss, R., \& Claeys, J. S. W. 2011, ApJ, 738, L1

Dilday, B., Howell, D. A., Cenko, S. B., et al. 2012, Sci, 337, 942

Elias-Rosa, N., Benetti, S., Cappellaro, E., et al. 2006, MNRAS, 369, 1880

Fitzpatrick, E. L. 1999, PASP, 111, 63

Fitzpatrick, E. L., \& Massa, D. 1990, ApJS, 72, 163

Folatelli, G., Morrell, N., Phillips, M. M., et al. 2013, ApJ, 773, 53

Foley, R. J., Fox, O. D., McCully, C., et al. 2014, MNRAS, 443, 2887

Förster, F., González-Gaitán, S., Folatelli, G., \& Morrell, N. 2013, ApJ, 772, 19

Garavini, G., Aldering, G., Amadon, A., et al. 2005, AJ, 130, 2278
Garavini, G., Folatelli, G., Goobar, A., et al. 2004, AJ, 128, 387

Garavini, G., Folatelli, G., Nobili, S., et al. 2007a, A\&A, 470, 411

Garavini, G., Nobili, S., Taubenberger, S., et al. 2007b, A\&A, 471, 527

Gerardy, C. L. 2005, in ASP Conf. Ser. 342, 1604-2004: Supernovae as Cosmological Lighthouses, ed. M. Turatto, S. Benetti, L. Zampieri \& W. Shea, 250

Gilfanov, M., \& Bogdán, Á 2010, Natur, 463, 924

Goldhaber, G., Groom, D. E., Kim, A., et al. 2001, ApJ, 558, 359

Gómez, G., \& López, R. 1998, AJ, 115, 1096

Goobar, A. 2008, ApJ, 686, L103

Goobar, A., Kromer, M., Siverd, R., et al. 2014, e-print (arXiv:1410.1363)

Guy, J., Astier, P., Nobili, S., Regnault, N., \& Pain, R. 2005, A\&A, 443, 781

Hamuy, M., Phillips, M. M., Suntzeff, N. B., et al. 2003, Natur, 424, 651

Hayden, B. T., Garnavich, P. M., Kasen, D., et al. 2010, ApJ, 722, 1691

Henyey, L. G., \& Greenstein, J. L. 1941, ApJ, 93, 70

Horesh, A., Kulkarni, S. R., Fox, D. B., et al. 2012, ApJ, 746, 21

Hsiao, E. Y., Conley, A., Howell, D. A., et al. 2007, ApJ, 663, 1187

Johansson, J., Woods, T. E., Gilfanov, M., et al. 2014, MNRAS, 442, 1079

Jones, D. O., Rodney, S. A., Riess, A. G., et al. 2013, ApJ, 768, 166

Justham, S. 2011, ApJ, 730, L34

Kim, A. G., Thomas, R. C., Aldering, G., et al. 2013, ApJ, 766, 84

Kromer, M., Sim, S. A., Fink, M., et al. 2010, ApJ, 719, 1067

Leonard, D. C. 2007, in AIP Conf. Ser. 937, Supernova 1987A: 20 Years After: Supernovae and Gamma-Ray Bursters, ed. S. Immler, K. Weiler \& R. McCray, 311

Li, W., Bloom, J. S., Podsiadlowski, P., et al. 2011, Natur, 480, 348

Li, W., Filippenko, A. V., Gates, E., et al. 2001, PASP, 113, 1178

Lira, P. 1995, PhD thesis, Masters Thesis, Universidad de Chile

Lundqvist, P., Mattila, S., Sollerman, J., et al. 2013, MNRAS, 435, 329

Maguire, K., Sullivan, M., Patat, F., et al. 2013, MNRAS, 436, 222

Mandel, K. S., Narayan, G., \& Kirshner, R. P. 2011, ApJ, 731, 120

Maoz, D., \& Mannucci, F. 2012, PASA, 29, 447

Nugent, P. E., Sullivan, M., Cenko, S. B., et al. 2011, Natur, 480, 344

O’Donnell, J. E. 1994, ApJ, 422, 158

Patat, F. 2005, MNRAS, 357, 1161

Patat, F., Benetti, S., Cappellaro, E., et al. 1996, MNRAS, 278, 111

Patat, F., Benetti, S., Cappellaro, E., \& Turatto, M. 2006, MNRAS, 369, 1949

Patat, F., Chandra, P., Chevalier, R., et al. 2007, Sci, 317, 924

Perlmutter, S., Gabi, S., Goldhaber, G., et al. 1997, ApJ, 483, 565

Phillips, M. M., Li, W., Frieman, J. A., et al. 2007, PASP, 119, 360

Phillips, M. M., Lira, P., Suntzeff, N. B., et al. 1999, AJ, 118, 1766

Phillips, M. M., \& Simon ????

Röpke, F. K., Kromer, M., Seitenzahl, I. R., et al. 2012, ApJ, 750, L19

Sako, M., Bassett, B., Becker, A. C., et al. 2014, e-print (arXiv:1401.3317)

Schlafly, E. F., \& Finkbeiner, D. P. 2011, ApJ, 737, 103

Schmidt, B. P., Kirshner, R. P., Leibundgut, B., et al. 1994, ApJ, 434, L19

Scolnic, D. M., Riess, A. G., Foley, R. J., et al. 2014, ApJ, 780, 37

Shappee, B. J., Stanek, K. Z., Pogge, R. W., \& Garnavich, P. M. 2013, ApJ, 762 , L5

Silverman, J. M., Ganeshalingam, M., \& Filippenko, A. V. 2013a, MNRAS, 430, 1030

Silverman, J. M., Nugent, P. E., Gal-Yam, A., et al. 2013b, ApJS, 207, 3

Silverman, J. M., Vinko, J., Kasliwal, M. M., et al. 2013c, MNRAS, 436, 1225

Sim, S. A., Fink, M., Kromer, M., et al. 2012, MNRAS, 420, 3003

Sim, S. A., Seitenzahl, I. R., Kromer, M., et al. 2013, MNRAS, 436, 333

Simon, J. D., Gal-Yam, A., Gnat, O., et al. 2009, ApJ, 702, 1157

Soker, N. 2014, MNRAS, 444, L73

Stanishev, V., Goobar, A., Benetti, S., et al. 2007, A\&A, 469, 645

Sternberg, A., Gal-Yam, A., Simon, J. D., et al. 2011a, Sci, 333, 856

Sternberg, A., Gal-Yam, A., Simon, J. D., et al. 2011b, Sci, 333, 856

Sternberg, A., Gal-Yam, A., Simon, J. D., et al. 2014, MNRAS, 443, 1849

Wang, L. 2005, ApJ, 635, L33

Wang, X., Li, W., Filippenko, A. V., et al. 2009, ApJ, 697, 380

Wang, X., Li, W., Filippenko, A. V., et al. 2008a, ApJ, 677, 1060

Wang, X., Li, W., Filippenko, A. V., et al. 2008b, ApJ, 675, 626

Welty, D. E., Ritchey, A. M., Dahlstrom, J. A., \& York, D. G. 2014, ApJ, 792, 106

Woods, T. E., \& Gilfanov, M. 2013, MNRAS, 432, 1640

Yamanaka, M., Naito, H., Kinugasa, K., et al. 2009, PASJ, 61, 713 